\title{
De l'avortement aux procréations artificielles, la toute-puissance du projet parental
}

\author{
Marie-Angèle Hermitte \\ Juriste, Centre de recherche en droit des sciences et des techniques, UMR8103, CNRS/Université Paris 1, 21bis rue Pasteur, \\ 92290 Malakoff, France
}

Luc Boltanski a livré en 2004 une somme monumentale sur la sociologie de l'avortement $^{1}$, sujet qui à première vue ne devrait pas intéresser les lecteurs de Natures Sciences Sociétés. Pourtant, dès l'introduction, l'auteur évoque une continuité entre l'avortement et les techniques de procréation, affirmant que «le développement des biotechnologies et particulièrement des techniques de la PMA (procréation médicalement assistée) aurait rencontré des difficultés considérables, si l'obstacle que constituait l'interdiction de l'avortement par rapport aux recherches sur la vie intra-utérine et sur l'embryon n'avait été levé »- dont acte. Le décor est posé, qui n'est pas sans rappeler celui qu'avait campé Michel Houellebecq dans Les Particules élémentaires ${ }^{2}$ : 1'avortement, pratique immémoriale, sa légalisation contemporaine de la «libération sexuelle», ont quelque chose à voir avec les biotechnologies. La logique qui amène de l'avortement aux techniques de procréation n'est expliquée clairement ni par le sociologue ni par le romancier. Mais, chez L. Boltanski, le fil rouge qui court, caché, dans le livre peut se retrouver: de l'avortement aux techniques de procréation, c'est la toute-puissance du «projet parental » qui fait le lien. Confrontée à une absence de projet parental ou, aussi bien, à un projet qui se révèle décevant, défaillant, une femme avorte. Animées par un projet parental que la nature refuse de servir, des personnes vont demander à la technique la réalisation de l'enfant rêvé. Je suggère que l'on s'attarde un instant sur la fresque

\footnotetext{
Auteur correspondant : mahermit@club-internet.fr

1 Boltanski, L., 2004. La Condition foetale : une sociologie de l'engendrement et de l'avortement, Paris, Gallimard.

2 Houellebecq, M. , 1998. Les Particules élémentaires, Paris, Flammarion. Le livre se termine en science-fiction sur l'apaisement de la société grâce à la reproduction par clonage ; c'était deux ans après l'annonce de la naissance de Dolly.
}

historique peinte par L. Boltanski, avant de revenir au cœur du projet parental et de ses conséquences.

\section{La fresque de L. Boltanski}

Tout démarre des «invariants » que L. Boltanski tire de l'étude comparative des pratiques d'avortement dans plusieurs centaines de sociétés, qui avait été réalisée par George Devereux, l'inventeur de l'ethnopsychiatrie, auteur d'un travail central souvent oublié sur le caractère universel et transhistorique de l'avortement ${ }^{3}$. Il en ressortait que l'avortement est une pratique fréquente, dont la possibilité est connue partout, qui fait l'objet d'une réprobation générale balancée par une tolérance. La tension entre les deux impose de trouver des solutions. Elles auront un caractère officieux, caché, encore aujourd'hui où l'on ne parle d'un avortement qu'à un très petit nombre de personnes. L'avortement n'est que très rarement « représenté », construit socialement. On ne le «parle» pas en dehors de l'hôpital, on ne le ritualise pas, ni pour marquer la délivrance ni pour marquer la tristesse ${ }^{4}$.

À partir de ces faits non contestables, L. Boltanski propose une hypothèse paradoxale : la possibilité de l'avortement est une condition nécessaire pour "créer des êtres humains nouveaux venant prendre place dans un monde peuplé par des vivants déjà là et habité par le souvenir des morts » (p. 42). En effet, accueillir de nouveaux êtres en leur donnant une place dans la société en tant qu'individus singularisés relève de mécanismes

\footnotetext{
${ }^{3}$ Devereux, G., 1955. A Study of Abortion in Primitive Societies, New York, International Universities Press.

4 Présenté ainsi, le propos est excessif. Les légendes d'âmes errantes des fœetus sont fréquentes, des autels sont dédiés aux fotus avortés au Japon, des rituels de purification après «fausses couches » ont existé partout.
} 
sociaux complexes qui n'ont rien à voir avec le fait de produire des enfants par la sexualité, mais avec l'inscription du nouvel être dans des rapports de reconnaissance mutuelle. Si l'enfant, une fois né, n'a pas de place suffisante dans son environnement social, alors l'avortement a toujours été considéré comme la moins mauvaise solution pour lui, pour la femme, pour le corps social. C'est la femme qui apprécie en dernière instance si les conditions de cette mise dans le monde social sont réunies. Pour autant, ce pouvoir apparemment exorbitant est étroitement encadré socialement par des instances extérieures, autant aujourd'hui qu'hier, malgré la légalisation.

Car telle est la seconde étape du raisonnement : L. Boltanski considère que l'universalité de la pratique de l'avortement montre que le fœetus, qui relève de l'espèce humaine par la chair, n'est pas, de ce seul fait, humain au sens où il serait engagé dans des liens sociaux. Il doit pour ce faire, être consacré par une parole d'accueil que seule la mère peut prononcer (p. 64). Il faut être "confirmé » pour devenir humain (p. 70), pour passer de la chair, vue comme une matière non instituée totalement substituable, à la personne reconnue dans des liens sociaux et juridiques ${ }^{5}$. La femme a ce pouvoir de "confirmation", curieux vocabulaire qui renvoie au sacré et à une conception évidemment dualiste de l'humanité.

Ayant terminé le deuxième mouvement de sa pensée sur cette conception tragique de l'engendrement comme évitement de l'avortement, il va développer, dans le troisième mouvement, les mécanismes qui permettent à une société donnée de faire en sorte que le plus grand nombre des fœetus produits par la sexualité soient des fœetus qu'il sera évident de confirmer par la parole. Ces mécanismes de «préconfirmation » des fœtus ne sont autres que les règles qu'une société se donne pour que la plupart des rapports sexuels donnent lieu à des fotus pour lesquels la question de la confirmation ne se posera pas. Il en va ainsi, évidemment, du rapport sexuel conjugal, qui rendra un enfant légitime socialement du simple fait du mariage de ses géniteurs. L'extravagante puissance de la mère est donc, en fait, soumise à une autorité extérieure qui reporte en amont de la mère l'opération de confirmation (p. 93). En dépossédant la mère de son pouvoir, la règle sociale la soulage du tragique de sa décision ; $c^{\prime}$ est ce que L. Boltanski appelle un «arrangement», qui permet de résoudre la contradiction entre la réprobation et la tolérance. Se faisant alors historien, il en donne trois exemples.

\footnotetext{
${ }^{5}$ On peine à suivre L. Boltanski jusqu'au bout de son raisonnement sur la substituabilité totale des chairs non instituées : " par la sexualité, les êtres détruits sont rapidement remplacés » (p. 71, 73). En effet, même les avortements totalement assumés laissent fréquemment à l'être détruit une place imaginaire dans l'histoire familiale : «il aurait 25 ans », «je suis sûre que c'était une fille», etc.
}

Le premier concerne un « arrangement spirituel avec le créateur ». Dans ce modèle de l'Occident chrétien, tous les fœetus de chair sont voulus par Dieu, interdisant en principe leur avortement. Mais, dans les premiers mois de la grossesse, le fœtus est un objet flou dans les consciences populaires - certaines femmes n'accouchentelles pas parfois d'objets étranges ${ }^{6} ?-$, objet flou aussi pour le monde savant, comme en témoigne la querelle de l'animation. On n'avortera pas, mais on fera " revenir les règles ", tel est un arrangement pensable - et largement pratiqué.

Un deuxième arrangement, avec la parenté, double le premier dans la même région du monde. Dans une société qui se donne pour objectif de ne reconnaître que les individus légitimés par le mariage de leurs parents, les stratégies patrimoniales vouent au célibat de nombreux individus dont l'activité sexuelle va produire des fœtus inexistants socialement, qui peuvent donc être facilement supprimés. Si l'avortement n'a pas lieu, c'est l'enfant qui, mis en nourrice ou dans des orphelinats, subira une mortalité infantile bien plus considérable que la moyenne, ou deviendra, s'il passe le cap, cette chair substituable que l'on envoie à la guerre, dont on fera des journaliers ou des valets, immense réservoir de main-d'œuvre servile, produite en permanence et totalement substituable.

Le troisième arrangement décrit par L. Boltanski, plus récent, tiendrait à l'emprise de l'État industriel. Celuici va s'intéresser au nombre et à la qualité des enfants produits. Ce projet, qu'il soit inspiré de Malthus ou des états eugénistes qui s'essaient à produire des citoyens de qualité par la stérilisation des porteurs de tares, des « trop pauvres » et l'élimination des handicapés, a peiné à s'insérer durablement dans ce qui constitue l'espace privé des familles.

Que reste-t-il de tout cela aujourd'hui? L. Boltanski suggère à juste titre qu'aucun de ces arrangements n'a totalement disparu (p. 127). Mais, alors qu'il semble considérer que l'État a quasiment abandonné le projet de gérer rationnellement la quantité et la qualité de la population (p. 127), on peut douter de la véracité de la proposition : montrer la continuité du projet va nous permettre de commencer à lier techniques et avortement. En effet, ce modèle de contrôle de la qualité et de la quantité des naissances est, en fait, aujourd'hui le modèle dominant, quoiqu'il se donne sous des formes renouvelées où l'État n'impose pas, mais organise et propose sans brutalité. Il n'y avait d'ailleurs aucune raison pour que le modèle eugéniste disparaisse, car il était possible d'écarter la brutalité politique du projet tout en laissant agir les volontés individuelles sur des bases scientifiques qui ont progressé. C'est donc à travers l'organisation du diagnostic prénatal, des enquêtes génétiques dans les familles à risques, et

\footnotetext{
${ }^{6}$ Darmont, P., 1981. Les Mythes de la procréation à l'âge baroque, Paris, Le Seuil.
} 
surtout à moyen terme de l'extension du diagnostic préimplantatoire d'embryons réalisés in vitro (DPI) que le projet s'exprime sous une forme renouvelée. Le DPI, qui a à peine une quinzaine d'années d'existence, est resté un temps focalisé sur des pathologies graves et rapidement mortelles, mucoviscidose ou myopathies. Mais, depuis deux ans, il s'intéresse aux risques de cancers familiaux et, récemment, un diagnostic génétique préimplantatoire d'un strabisme vient d'être autorisé en Grande-Bretagne par la Haute Autorité britannique pour la fertilisation et l'embryologie humaines ${ }^{7}$. Le contrôle biologique de la qualité des naissances gagne sans cesse en puissance, car les objectifs de l'État, des parents, des médecins et des biologistes sont très proches ${ }^{8}$. Si, pour l'État, il s'agit de façonner une population de bonne qualité sur un plan collectif, pour les parents, il s'agit d'avoir des enfants qui correspondent à leur projet parental. Quel est, alors, le lien entre ce que ces techniques disent de la société et l'avortement?

L. Boltanski répond en montrant que, dans la fresque historique de l'avortement, la période contemporaine a inventé un nouveau type "d'arrangement». Il s'agit de ne mettre au monde que les fotus qui correspondent au «projet parental» actuel. Nombre de femmes qui avortent ne sont pas confrontées aux difficultés traditionnelles: poids de la société ou de l'Église sur les «bâtards », impossibilité économique, trop grand nombre d'enfants dans la famille, etc. Non, ces femmes vont avorter parce que leur partenaire, le couple qu'elles forment avec lui ne donnera pas le bon cadre pour élever un enfant et en faire une personne : le fotus produit par le rapport sexuel n'est pas le sujet d'un projet parental de bonne qualité, il ne sera pas confirmé.

Pour arriver à cette conclusion, L. Boltanski s'appuiera sur les spécificités d'une sous-population représentative de la modernité : femmes urbaines confrontées

\footnotetext{
7 BBC News, 8 mai 2007, http://news.bbc.co.uk/2/hi/health/ 6634015.stm ;cité sur http://www.genethique.org/revues/ revues/2007/mai/20070515.3.asp. Sur les risques de dérapage du DPI, cf. Testart, J. , 1994. Le Désir du gène, Paris, Flammarion.

8 Les objectifs peuvent toutefois diverger, par exemple en Inde et en Chine où l'avortement des fœetus féminins a d'ores et déjà provoqué un déséquilibre important du sex ratio dans certaines classes sociales urbaines. Les États cessent alors d'avoir les mêmes stratégies que les familles, faisant renaître un conflit ; il faut noter aussi que les stratégies privées des couples continuent d'apporter des démentis à l'efficacité du modèle. Ainsi, si beaucoup de fœetus trisomiques sont effectivement détectés, le nombre de personnes trisomiques baisse assez peu, du fait de l'augmentation du nombre de grossesses tardives, choix personnel. De même, les stratégies parentales et judiciaires visant à obtenir des indemnisations lorsque l'anomalie n'a pas été repérée ont conduit à une augmentation considérable des primes d'assurance des échographistes et à l'abandon de cette pratique par nombre d'entre eux, ce qui devrait se traduire à court terme par l'augmentation du nombre de naissances d'enfants handicapés.
}

à des vies affectives précaires, ayant souvent avorté deux ou trois fois alors qu'elles connaissent bien les règles de la contraception. L'accident de contraception est d'ordre comportemental plutôt que technique et laisse souvent pointer un certain désir de grossesse dont la femme est consciente. Les paroles qui vont s'échanger au moment de l'annonce de la grossesse au partenaire, la réaction du père, vont décider en un court espace de temps du sort du fœetus. Si le père montre son aptitude à endosser un projet de vie et d'éducation, le fœtus sera confirmé, il ne le sera pas dans le cas contraire, car la relation sexuelle n'aura pas débouché sur la construction du couple parental jugé indispensable à l'éducation de l'enfant.

Le concept de projet parental, ambivalent, a vocation à structurer aussi bien l'avortement que l'engendrement, qui sont les deux faces d'un même phénomène : exclure ou accueillir dans le monde un nouvel être, opérations universelles et immémoriales qui se construisent selon divers modèles historiques, que L. Boltanski va illustrer et analyser.

\section{Le projet parental, nouveau type d'arrangement pour l'avortement}

La légalisation de l'avortement aurait dû supprimer l'ancienne tension entre interdit et tolérance. De même, la disponibilité des techniques de contraception apparaissait comme un moyen radical de ne concevoir que des fœtus voulus, donc préconfirmés par la femme elle-même, en dehors de toute instance sociale et sans nécessité d'arrangement : la technique aurait dû effacer la nécessité des arrangements. Pourtant, les "échecs de contraception ", nombreux, sont de nature plus souvent comportementale que technique (p. 146, p. 159 : un exemple très parlant dans lequel l'amant évoque son désir d'avoir un enfant de cette femme, ce qui l'émeut alors qu'elle n'est pas prête pour en avoir, et coïncide avec une pratique de contraception moins rigoureuse). Donner un cadre juridiquement reconnu au pouvoir de sélection des femmes n'a pas suffi à supprimer la tension. L'avortement reste un sujet dénié et les arrangements restent nécessaires pour dénouer la tension : il faut toujours justifier le geste. Désormais détentrices d'un pouvoir légal de sélection, les femmes ne semblent toujours pas décider seules, en fonction de leur désir personnel, d'avoir un enfant et continuent de référer leur décision à une instance extérieure, en l'occurrence l'image du couple parental, et plus particulièrement du type de père nécessaire pour faire du fœetus de chair une «vraie personne» (p. 131).

Car la nouvelle contrainte extérieure, qui conduit à pérenniser la sélection des fotus, est la nécessité ressentie de ne faire naître des enfants que s'il est possible de leur donner un cadre familial où ils pourront s'épanouir, soutenus par des pères responsables, "matures ", 
qui assument avec les mères un projet de vie pour l'enfant. En somme, aux temps précaires qui sont les nôtres, où les structures traditionnelles de légitimation des liens familiaux ont perdu de leur force, où un mariage sur trois se termine par un divorce, certaines femmes opposent un rêve de perfection familiale. Si le père reçoit l'annonce de la grossesse d'une manière qui fait douter de son engagement, s'il se retranche derrière la formule consacrée : «C'est à toi de décider» (p. 153), s'il n'est qu'une aventure qui ne permet pas à la femme de se « projeter dans l'avenir » (p. 133), si la mère, engagée dans une relation avec deux hommes, ne sait pas qui est le père, si elle ne peut pas «admirer » le père (p. 134), si celui-ci ne paraît pas " concerné », intéressé (p. 151), alors le fœetus risque fort de ne pas être confirmé, car l'enfant serait voué à ce malheur moderne de ne pas être un «projet d'enfant » (p. 149, $169)^{9}$. La grossesse non voulue devient une «épreuve» dont le couple doit sortir vainqueur pour que le fœetus, mis en jeu au sens propre du terme, soit confirmé. Si l'image du projet parental renvoyée par le couple n'est pas l'image idéale, alors le fœtus doit être supprimé, car mettre au monde est un acte grave, tandis que l'élimination d'un fœetus est l'élimination d'un rien, on le verra.

Effectivement, dans le chapitre V, L. Boltanski étudie la «construction » des catégories fœtales, soit les multiples sortes d'embryons et de fœetus qui se dégagent du discours que l'on tient sur eux, en considération de la situation dans laquelle ils se trouvent ${ }^{10}$. Car, dans le discours, il n'existe pas de fœetus en tant que tel, hormis dans l'instance de la religion, mais des « sortes de fœetus » dont le corps concret n'a pas d'autre réalité que celle de la qualification qui lui est donnée et qui, elle, dépend du projet parental.

Le fœtus «non confirmé » est, ainsi, qualifié de fœtus tumoral (expression de L. Boltanski et, d'ailleurs, de certains médecins, mais qui n'est pas employée par les femmes, qui parlent du rien ou presque rien, du non-être, du truc, du ça, de la molécule chimique, etc.). Il faut l'extraire en laissant le moins de traces possible. Pris dans le processus hospitalier de l'IVG, il ne sera pas nommé, raconté, visualisé. On ne le montre pas à l'échographie, on l'expulse dans une « chaussette » qui permet de ne jamais

\footnotetext{
${ }^{9}$ L. Boltanski a peut-être présenté ce modèle de manière trop abrupte, car le lecteur pourrait avoir le sentiment qu'il est généralisé. Or, s'il est très fort, il est contredit par d'autres tendances elles aussi puissantes, comme la volonté de nombre de femmes d'avoir un ou des enfants malgré l'absence du père, ce qui est un projet parental à part entière et explique pour partie la multiplication des familles monoparentales.

${ }_{10}$ La manipulation des catégories fœtales par la médecine a fait l'objet de travaux déjà anciens : cf. Oliviero, P., 1991. La notion de pré-embryon dans la littérature politico-scientifique, Archives de philosophie du droit, 36, 85-107 ; Hermitte, M-A., 1990. L'embryon aléatoire, in Testart, J. (Ed.), Le Magasin des enfants, Paris, François Bourin, 238-265 (rééd. Paris, Gallimard, 1994, 327-367).
}

le voir jusqu'à l'incinérateur des déchets ${ }^{11}$. Il s'oppose au fœutus authentique, que l'on regarde à l'échographie, auquel on donne un prénom, que l'on appelle volontiers « le bébé »; il faudrait ajouter que, s'il meurt avant terme, des parents intentent des actions en justice, perdues, pour homicide involontaire, réclament une sépulture, une place à l'état civil ${ }^{12}$.

À ces deux catégories, « construites » par la possibilité de l'avortement, s'ajoutent les catégories construites par l'instance technique, les techno-fœetus, fabriqués par la technique. Les embryons conçus in vitro, bien sûr, mais aussi le fœetus aimé qui, à la suite d'un examen de routine, est reconnu atteint d'une maladie grave et incurable, et bascule brutalement du côté de la suppression. Pourtant, il est dans un territoire flou : il n'est pas du rien comme le fœtus tumoral, il subira autant que faire se peut une «mort douce » et les cas de demande de sépulture sont fréquents. Il aura souvent reçu un nom et les parents auront subi la perte d'un «enfant». Le projet parental existait pour le préconfirmer, mais il concernait l'enfant en pleine santé du projet hygiéniste : le corps handicapé dont cet enfant a été doté $\mathrm{n}^{\prime}$ est pas conforme au projet ${ }^{13}$. Logique, donc - comme l'avait montré Marcela Iacub ${ }^{14}$, dont les raisonnements sont repris par L. Boltanski -, de permettre à l'enfant né handicapé de demander la réparation de son handicap. Dans ce modèle, celui qui plaide est, en tant que sujet de droit, l'enfant abstrait que ses parents avaient doté d'un corps parfait et qui demande réparation de n'avoir pas reçu le corps prévu. Parce que les parents n'ont pas été informés d'un handicap du corps fotal quand il était temps de l'effacer par l'avortement, il n'a pas été possible d'en substituer un autre en bonne santé. Dans ce curieux schéma, le sujet a droit au corps du projet parental, car le corps concret est un « accident » au sens aristotélicien; la personne rêvée est la seule substance qui a droit au corps rêvé, puisque celui-ci est constructible par la technique, toujours substituable : les corps fœtaux ne sont que des brouillons et $\mathrm{l}^{\prime}$ on peut recommencer jusqu'à obtenir le bon corps pour ce projet d'enfant qui, lui, reste unique. Jusque-là, que l'on soit d'accord ou pas avec cette métaphysique radicale, les choses paraissent claires, il y a projet ou pas, la réalité est conforme au projet ou pas. Mais où s'arrête

\footnotetext{
${ }^{11}$ Ce qui cesse d'être vrai avec l'avortement médicamenteux, posant problème à certaines femmes et au personnel hospitalier.

12 Lavigne, C., et al., 2006. Pratiques hospitalières concernant les foetus mort-nés et nouveau-nés décédés : CHU de Paris, Lyon et Marseille. Rapport de synthèse, Inspection générale des affaires sociales, n 2006024 / Inspection générale de l'administration de l'Éducation nationale et de la Recherche, $n^{\circ}$ 2006-016, Paris.

${ }^{13} \mathrm{Cf}$. un cas américain atypique où un couple d'homosexuelles sourdes a conçu le projet de mettre au monde un enfant atteint de la même surdité génétique. Il fallut trouver le sperme d'un homme atteint de la même maladie. L'enfant naquit effectivement sourd.
}

14 Iacub, M., 2002. Penser les droits de la naissance, Paris, PUF. 
la logique de projet? Car, si on y entre, il est difficile de justifier une limite plutôt qu'une autre, et c'est peut-être ce que L. Boltanski ne pousse pas à son terme, pas plus que ne l'avait fait $\mathrm{M}$. Iacub.

\section{Car le projet parental est multiple... et peut cesser d'être parental}

Restons en premier lieu dans le projet parental en essayant de le décliner. Le projet parental auquel on pense d'emblée est conçu entre deux personnes vivantes, liées par une relation affective ayant un minimum de connotation sexuelle. La vision la plus restrictive est celle de la loi française qui limite l'accès à la procréation assistée aux couples hétérosexuels vivants, en âge de procréer. Nul doute que ces limites céderont rapidement. Le projet d'enfant peut animer une femme ou un homme seuls, un individu séparé de son conjoint, ou dont le conjoint est décédé mais qui veut pouvoir disposer des embryons, des couples homosexuels, des couples âgés. Toutes sortes de projets parentaux existent donc et, en dehors des convictions personnelles, il est difficile de décider en raison qu'un projet est valable et l'autre pas. Ouvrir la procréation aux couples homosexuels dans le cadre d'une égalité en droit totale implique de leur permettre comme à tout un chacun la transmission de leurs patrimoines génétiques, ce qui oblige à accepter le clonage reproductif et les mères porteuses. Le concept de projet parental a donc une forte dynamique interne, qui fait obstacle à l'établissement de limites rigoureusement justifiées.

Peut-on aller plus loin? Il serait assez tendance de concevoir des enfants entre personnes ayant un projet abstrait commun, mais aucun lien physique, l'un en Asie, l'autre aux États-Unis; les gamètes se rencontreront dans une zone franche... Internet peut permettre cela sans grande difficulté. Allons plus loin : au nom de quoi limiter la conception intellectuelle d'un projet parental aux personnes physiques? Pourquoi, dans un monde qui devrait être atteint à moyen terme par une baisse démographique généralisée, refuser à une personne morale de faire des projets d'enfants, élevés par des salariés pour continuer de peupler le monde et entretenir les vieux? Les Lebensborn du national-socialisme ${ }^{15}$ ou les enfants collectifs des kibboutz ${ }^{16}$ étaient faits de manière archaïque, mais l'idée d'un projet parental collectif, pensé par des personnes morales, était bien là. Il a échoué et certains procès en dommages-intérêts sont en cours...

Plus près de nous dans le temps, quittons le monde $\mathrm{du}$ projet parental et restons dans la logique du projet,

\footnotetext{
${ }_{15}$ Hillel, M., 1975. Au nom de la race, Paris, Fayard.

16 Bettelheim, B., 1971. Les Enfants du rêve, Paris, Robert Laffont.
}

non plus parental, mais scientifique cette fois. Car l'un ne va pas sans l'autre. Le monde scientifique, s'appuyant depuis le début de la révolution procréatique sur la légalisation de l'avortement, a en effet cherché à obtenir un pouvoir de suppression de même nature. Le fait que les femmes puissent avorter n'est pas analysé par eux comme l'effet d'une relation tragique, mais comme la preuve que le fœtus est une entité sans valeur s'il est désinvesti. Dès lors, les médecins peuvent expérimenter sur les embryons désinvestis par le projet parental initial ${ }^{17}$. Et comme ces embryons ou fœtus désinvestis ne sont pas assez nombreux ni assez spécialisés pour les différents projets scientifiques, alors il est plus rationnel de créer des embryons à des fins exclusivement scientifiques et désormais thérapeutiques. La Grande-Bretagne a donc depuis longtemps légalisé la possibilité de concevoir des embryons à d'autres fins qu'un projet parental, sur un projet scientifique. Il est possible de créer des embryons spécialement destinés à la recherche scientifique et, désormais, il est également légal de créer, par transfert de noyau d'une cellule somatique dans un ovocyte énucléé, des embryons uniquement destinés à fournir des cellules souches embryonnaires. Pour justifier cette possibilité, bien des effets de langage et de catégories ont été utilisés. Henri Atlan nie qu'un transfert de noyau donne un embryon humain, puisqu'il n'est pas issu d'une fécondation. Ce serait "autre chose », même si cette chose placée dans un utérus est susceptible de donner un enfant. On va donc parler d'embryons dépourvus de projet parental, mais aussi de préembryons, de cellules embryonnaires, ou de transfert de noyau pour éviter le terme clonage thérapeutique, qui marque trop la proximité avec le clonage reproductif et l'identité de l'entité qui deviendrait un enfant dans un cas, un médicament dans l'autre. Il serait plus honnête de parler d'embryons soutenus par un projet scientifique. Certains proposent même de n'appeler embryon que celui qui est replacé dans l'utérus d'une femme, faisant des embryons in vitro de simples choses. Le corps embryonnaire n'a donc aucune existence en soi, il n'est qu'un support de projets. La boucle est ainsi bouclée, du projet parental unique aux projets parentaux de toutes sortes, puis aux multiples projets scientifiques et industriels.

Ce glissement de l'un vers les autres n'a, en fait, rien d'étonnant, car le concept de projet parental, latent dans la société en raison du vieux rêve de maîtrise de la procréation, a tout de même été construit et nommé comme tel par les médecins pratiquant la PMA et les comités d'éthique, pour les besoins de l'encadrement de l'accès aux techniques de procréation. S'il a été utilisé pour interdire les couples homosexuels ou les femmes ménopausées ou pour encadrer le sort des embryons, « soutenus

\footnotetext{
17 Cf. les raisonnements du médecin et député Palacios, cité
} dans les articles de P. Oliviero et M-A. Hermitte (supra, note 10). 
par un projet parental » et conservés, ou « dépourvus de projet parental » et pouvant être détruits, il peut aussi bien être utilisé en sens inverse, comme on l'a montré, car le contenu du projet est par hypothèse malléable, soumis à l'expression de la volonté individuelle, du projet politique ou de la dynamique économique. On peut, pour les besoins de chaque cause, créer à partir d'un substrat corporel unique, le corps embryonnaire, une multitude de catégories que l'on investira librement comme sujets ou comme objets.

La loi française semblait opposer un obstacle de taille à l'industrialisation du corps embryonnaire, dans la mesure où l'article $16 \mathrm{du}$ Code civil affirme que la loi «garantit le respect de l'être humain dès le commencement de sa vie ", respect mesuré, distinct de la dignité, applicable à la seule personne. En distinguant deux régimes, celui de la personne née et celui de l'être humain non né, le Code entendait signifier que les embryons n'avaient pas les droits de la personne et particulièrement pas le droit à la vie, mais qu'ils devaient être traités de manière respectueuse. Les travaux parlementaires montraient que ce respect avait été entendu comme un obstacle posé à leur « réification ». Pour accéder au clonage thérapeutique, il faut précisément pouvoir réifier cet embryon pour en faire un médicament. L'obstacle de la loi n'est dirimant qu'en apparence, puisqu'il suffit de dire que l'entité issue du clonage thérapeutique n'est pas un embryon, mais l'objet d'un projet thérapeutique. Les choses sont faciles à manipuler. Pourtant, entre la destruction de l'embryon par la femme qui le porte, relation de mort entre deux individus confrontés involontairement l'un à l'autre, et la création d'un embryon pour en faire un objet thérapeutique, il y a une distance incommensurable. Curieusement, L. Boltanski ne semble pas s'intéresser à cette différence, alors qu'il s'agit bien d'une rupture radicale que la science, par une captation magistrale, a justifiée par la revendication des femmes. Giorgio Agamben et son travail sur la vie nue, la chair humaine sans protection, totalement désinstituée, sont cités dans la bibliographie, mais pas utilisés ${ }^{18}$. Or, le projet scientifique du clonage thérapeutique est la création d'un nouveau type de vies nues, sans douleur, sans conscience et sans parole.

\footnotetext{
18 Agamben, G., 1997. Homo sacer. I, Le Pouvoir souverain et la vie nue, Paris, Le Seuil ; cf. le très important article intitulé « Forme-
} de-vie », mis en ligne en janvier 1993 sur le site de la revue Multitudes : http://multitudes.samizdat.net/article673.html 